\title{
Further results on the group inverse of some anti-triangular block matrices
}

\author{
Chongguang Cao • Hanyu Zhang • Yanling Ge
}

Received: 25 September 2013 / Published online: 30 November 2013

(C) The Author(s) 2013. This article is published with open access at Springerlink.com

\begin{abstract}
Suppose $\mathfrak{R}$ is a right Ore domain with unity 1 . In this paper, we investigate the existence of the group inverse of some anti-triangular block matrices over $\Re$ and obtain the sufficient and necessary conditions for such existence. Further, the representations of the group inverse for the following two classes are given.

(i) $M=\left(\begin{array}{ll}A & B \\ C & 0\end{array}\right)$, where $C A=C$;

(ii) $M=\left(\begin{array}{cc}A & B \\ B & 0\end{array}\right)$, where $B^{\sharp}$ exists and $B A=B A B^{\sharp} B$.

The results extend the earlier works of Liu et al. (in Appl. Math. Comput. 218:8978-8986, 2012) and Zhao et al. (in E. J. Linear Algebra 21:63-75, 2010). Some results in special cases are also generalized to any ring.
\end{abstract}

Keywords Right Ore domain · Block matrix · Group inverse · Ring

Mathematics Subject Classification $15 \mathrm{~A} 09 \cdot 16 \mathrm{U} 30 \cdot 65 \mathrm{~F} 20$

Supported by National Natural Science Foundation of China (NO. 11371109).

C. Cao $(\bowtie) \cdot H$. Zhang · Y. Ge

School of Mathematical Science, Heilongjiang University, Harbin 150080, China

e-mail: caochongguang@163.com

H. Zhang

e-mail: zhanghanyu423@126.com

Y. Ge

e-mail: geyanling00@126.com 


\section{Introduction}

For a square matrix $A$, the matrix $X$ is called the group inverse of $A$ if $X$ satisfies the matrix equations

$$
A X A=A, X A X=X \quad \text { and } \quad A X=X A .
$$

It is well known that if the group inverse $X$ exists, it is unique, and is denoted by $A^{\sharp}$. Let $A^{\pi}$ be $I-A A^{\sharp}$.

The group inverse of block matrix is a very useful tool in many fields, such as iterative methods, Markov chains, singular differential and difference equations, see [1-8].

In 1983, in the context of differential equations, Campbell et al. [9] proposed the problem of finding the representation for the Drazin inverse (group inverse) of the anti-triangular block matrix $\left(\begin{array}{ll}A & B \\ C & 0\end{array}\right)$. This problem remains open. However, there are many results in some special cases, see [10, 13-18, 20-22]. It is important to study them in a larger ring, see for example $[11,12,19,26]$.

Liu et al. [10] studied this problem under the conditions $A^{2}=A, C A=C$ over complex fields. In this paper, we not only delete the condition $A^{2}=A$ but also solve it for the matrices over right Ore domains by using matrix equations. This also generalizes the results of Ge et al. [27].

On the other hand, in [20], Zhao et al. characterized the existence and the representation of group inverse for block matrix over skew fields $\left(\begin{array}{cc}A & B \\ B & 0\end{array}\right)$ under the condition that $B^{\sharp}$ exists and $B A B^{\pi}=0$. In this paper, we extend these results to right Ore domains. Some results in special cases are studied over rings with unity 1 .

In this paper, let $\mathbf{R}$ be a ring with unity 1. A ring is called a right Ore domain (denoted by $\Re$ ) if it possesses no zero divisors and every two elements of the ring have a right common multiple. Integral rings, polynomial rings in an indeterminate over field, noncommutative principal ideal domains and so on are right Ore domains. A left Ore domain is defined similarly. Every right (left) Ore domain $\Re$ can be embedded in the skew field (denoted by $K_{\Re}$ ) of quotients of itself. More details are found in [23-25]. Let $\Re^{m \times n}$ (respectively, $\mathbf{R}^{m \times n}$ ) be the set of all $m \times n$ matrices over $\Re$ (respectively, $\mathbf{R}$ ). The rank of a matrix $A \in \mathfrak{R}^{m \times n}$ (denoted by $\mathrm{r}(\mathrm{A})$ ) is defined as the rank of $A$ over $K_{\Re}$, i.e., the maximum order of all invertible subblocks of $A$ over $K_{\Re}$. For convenience, we suppose the right Ore domain $\Re$ has identity 1 .

\section{Some lemmas}

The following three lemmas will be used in the paper.

Lemma 1 [12] Let $A \in \Re^{n \times n}$. Then the following are equivalent:

(i) $A^{\sharp}$ exists;

(ii) $A^{2} X=A$ for some $X \in \Re^{n \times n}$. In this case, $A^{\sharp}=A X^{2}$;

(iii) $Y A^{2}=A$ for some $Y \in \Re^{n \times n}$. In this case, $A^{\sharp}=Y^{2} A$. 
Lemma $2[11,26]$ Let $A \in \mathbf{R}^{n \times n}$. Then the following are equivalent:

(i) $A^{\sharp}$ exists;

(ii) $A^{2} X=A, Y A^{2}=A$ for some $X, Y$ over $\mathbf{R}$. In this case, $A^{\sharp}=Y^{2} A=A X^{2}=$ $Y A X$.

Lemma 3 Let $A, B \in \mathbf{R}^{n \times n}$. If $B A B^{\pi}=0, B^{\sharp}$ and $\left(A B^{\pi}\right)^{\sharp}$ exist, then

(i) $B^{\sharp} A B^{\pi}=0,\left(A B^{\pi}\right)^{\sharp} B=0, B\left(A B^{\pi}\right)^{\sharp}=0$;

(ii) $A\left(A B^{\pi}\right)^{\sharp}=\left(A B^{\pi}\right)^{\sharp} A B^{\pi}$;

(iii) $A\left(A B^{\pi}\right)^{\sharp} A B^{\pi}=A B^{\pi}$.

Proof (i) $B^{\sharp} A B^{\pi}=\left(B^{\sharp}\right)^{2} B A B^{\pi}=0,\left(A B^{\pi}\right)^{\sharp} B=\left(\left(A B^{\pi}\right)^{\sharp}\right)^{2} A B^{\pi} B=0$, similarly, $B\left(A B^{\pi}\right)^{\sharp}=0$.

(ii) $A\left(A B^{\pi}\right)^{\sharp}=A B^{\pi}\left(A B^{\pi}\right)^{\sharp}=\left(A B^{\pi}\right)^{\sharp} A B^{\pi}$.

(iii) From (ii), we can easily show that (iii) holds.

\section{Main results}

The following is the main result in this note.

Theorem 1 Let $M=\left(\begin{array}{ll}A & B \\ C & 0\end{array}\right)$, where $A \in \Re^{n \times n}, B \in \Re^{n \times m}, C \in \Re^{m \times n}$. If $C A=C$, then

(i) $M^{\sharp}$ exists if and only if $(C B)^{\sharp}$ and $A^{\sharp}$ exist, $A^{\pi} B(C B)^{\pi}=0$;

(ii) If $M^{\sharp}$ exists, then $M^{\sharp}=\left(\begin{array}{ll}M_{1} & M_{2} \\ M_{3} & M_{4}\end{array}\right)$, where

$$
\begin{aligned}
& M_{1}=A^{\sharp}-\left(A^{\sharp}\right)^{2} B(C B)^{\pi} C-A^{\sharp} B(C B)^{\sharp} C-A^{\sharp} B(C B)^{\pi} C-A^{\pi} B\left[(C B)^{\sharp}\right]^{2} C, \\
& M_{2}=\left(A^{\sharp}\right)^{2} B(C B)^{\pi}+A^{\pi} B\left[(C B)^{\sharp}\right]^{2}+A^{\sharp} B(C B)^{\sharp}+A^{\pi} B(C B)^{\sharp}, \\
& M_{3}=(C B)^{\pi} C+(C B)^{\sharp} C, \\
& M_{4}=-(C B)^{\sharp} .
\end{aligned}
$$

Proof (i) The "only if" part.

Since $C A^{2}=C A=C$, we have

$$
\left(\begin{array}{ll}
I & B \\
0 & I
\end{array}\right)\left(\begin{array}{ll}
I & 0 \\
C & I
\end{array}\right)\left(\begin{array}{cc}
A^{2} & A B-B C B \\
0 & C B C B
\end{array}\right)=\left(\begin{array}{cc}
A^{2}+B C & A B \\
C & C B
\end{array}\right)=M^{2}
$$

By Lemma $1, M^{\sharp}$ exists if and only if $M=Y M^{2}$ for some $Y \in \Re^{(n+m) \times(n+m)}$. Let

$$
Y\left(\begin{array}{ll}
I & B \\
0 & I
\end{array}\right)\left(\begin{array}{ll}
I & 0 \\
C & I
\end{array}\right)=\left(\begin{array}{ll}
Y_{1} & Y_{2} \\
Y_{3} & Y_{4}
\end{array}\right)
$$

where $Y_{1} \in \mathfrak{R}^{n \times n}$, so

$$
\left(\begin{array}{ll}
Y_{1} & Y_{2} \\
Y_{3} & Y_{4}
\end{array}\right)\left(\begin{array}{cc}
A^{2} & A B-B C B \\
0 & C B C B
\end{array}\right)=\left(\begin{array}{ll}
A & B \\
C & 0
\end{array}\right)
$$

it follows that $Y_{1} A^{2}=A$, by Lemma 1 , so $A^{\sharp}$ exists. 
According to Lemma $1, M^{\sharp}$ exists if and only if there exists a matrix $X=$ $\left(\begin{array}{ll}X_{1} & X_{2} \\ X_{3} & X_{4}\end{array}\right) \in \Re^{(n+m) \times(n+m)}$ such that $M^{2} X=M$, where $X_{1} \in \mathfrak{R}^{n \times n}$.

By (1), we have

$$
\left(\begin{array}{ll}
I & B \\
0 & I
\end{array}\right)\left(\begin{array}{ll}
I & 0 \\
C & I
\end{array}\right)\left(\begin{array}{cc}
A^{2} & A B-B C B \\
0 & C B C B
\end{array}\right) X=M
$$

Hence,

$$
\left(\begin{array}{cc}
A^{2} & A B-B C B \\
0 & C B C B
\end{array}\right)\left(\begin{array}{ll}
X_{1} & X_{2} \\
X_{3} & X_{4}
\end{array}\right)=\left(\begin{array}{cc}
I & 0 \\
-C & I
\end{array}\right)\left(\begin{array}{cc}
I & -B \\
0 & I
\end{array}\right) M
$$

Namely,

$$
\begin{gathered}
A^{2} X_{1}+(A B-B C B) X_{3}=A-B C, \\
A^{2} X_{2}+(A B-B C B) X_{4}=B, \\
C B C B X_{3}=C B C, \\
C B C B X_{4}=-C B .
\end{gathered}
$$

From (5) and Lemma 1, we know $(C B)^{\sharp}$ exists. By (3), we have $-A^{\pi} B C B X_{4}=$ $A^{\pi} B$. i.e.,

$$
-A^{\pi} B(C B)^{\sharp} C B C B X_{4}=A^{\pi} B .
$$

Substitute (5) into the above equation, we have $A^{\pi} B(C B)^{\sharp} C B=A^{\pi} B$. Therefore, $A^{\pi} B(C B)^{\pi}=0$.

The "if" part.

Let $X_{1}=A^{\sharp}-A^{\sharp^{2}} B(C B)^{\pi} C-A^{\sharp} B(C B)^{\sharp} C, X_{2}=A^{\sharp^{2}} B(C B)^{\pi}+A^{\sharp} B(C B)^{\sharp}$, $X_{3}=(C B)^{\sharp} C, X_{4}=-(C B)^{\sharp}$.

Note that $A^{\pi} B(C B)^{\pi}=0$. It is easy to verify that (2)-(5) hold. This implies $M=$ $M^{2} X$ has a solution, so $M^{\sharp}$ exists.

(ii) By Lemma $1, A^{\pi} B(C B)^{\pi}=0$ and $C A^{\sharp}=C A A^{\sharp}=C A^{2} A^{\sharp}=C A=C$, the expression of $M^{\sharp}$ can be obtained from $M^{\sharp}=M X^{2}$. Next we can compute that

$$
\begin{aligned}
M^{\sharp}= & \left(\begin{array}{ll}
A & B \\
C & 0
\end{array}\right)\left(\begin{array}{ll}
X_{1} & X_{2} \\
X_{3} & X_{4}
\end{array}\right)\left(\begin{array}{ll}
X_{1} & X_{2} \\
X_{3} & X_{4}
\end{array}\right) \\
= & \left(\begin{array}{ll}
A & B \\
C & 0
\end{array}\right) \\
& \times\left(\begin{array}{cc}
A^{\sharp}-A^{\sharp^{2}} B(C B)^{\pi} C-A^{\sharp} B(C B)^{\sharp} C & A^{\sharp^{2}} B(C B)^{\pi}+A^{\sharp} B(C B)^{\sharp} \\
(C B)^{\sharp} C & -(C B)^{\sharp}
\end{array}\right) \\
& \times\left(\begin{array}{cc}
X_{1} & X_{2} \\
X_{3} & X_{4}
\end{array}\right) \\
= & \left(\begin{array}{cc}
A A^{\sharp}-A^{\sharp} B(C B)^{\pi} C+A^{\pi} B(C B)^{\sharp} C & A^{\sharp} B(C B)^{\pi}-A^{\pi} B(C B)^{\sharp} \\
(C B)^{\pi} C & C B(C B)^{\sharp}
\end{array}\right)
\end{aligned}
$$




$$
\begin{aligned}
& \times\left(\begin{array}{ll}
X_{1} & X_{2} \\
X_{3} & X_{4}
\end{array}\right) \\
= & \left(\begin{array}{cc}
A^{\sharp}-A^{\sharp^{2}} B(C B)^{\pi} C-A^{\sharp} B(C B)^{\sharp} C & A^{\sharp^{2}} B(C B)^{\pi}+A^{\pi} B\left((C B)^{\sharp}\right)^{2} \\
-A^{\sharp} B(C B)^{\pi} C-A^{\pi} B\left((C B)^{\sharp}\right)^{2} C & +A^{\sharp} B(C B)^{\sharp}+A^{\pi} B(C B)^{\sharp} \\
(C B)^{\pi} C+(C B)^{\sharp} C & -(C B)^{\sharp}
\end{array}\right) \\
= & \left(\begin{array}{cc}
M_{1} & M_{2} \\
M_{3} & M_{4}
\end{array}\right) .
\end{aligned}
$$

Example for Theorem 1: Let $\Re$ be the integer ring, and let $M=\left(\begin{array}{ll}A & B \\ C & 0\end{array}\right)$, where

$$
A=\left(\begin{array}{ccc}
1 & 0 & 0 \\
0 & -1 & 0 \\
0 & 0 & 0
\end{array}\right), \quad B=\left(\begin{array}{ll}
1 & 0 \\
1 & 3 \\
2 & 0
\end{array}\right), \quad C=\left(\begin{array}{ccc}
1 & 0 & 0 \\
2 & 0 & 0
\end{array}\right) \text {. }
$$

It is easy to verify $A^{2} \neq A, C A=C$. Furthermore, $A^{\sharp}$ and $(C B)^{\sharp}$ exist.

By computation,

$$
\begin{aligned}
& A^{\sharp}=\left(\begin{array}{ccc}
1 & 0 & 0 \\
0 & -1 & 0 \\
0 & 0 & 0
\end{array}\right), \quad A^{\pi}=\left(\begin{array}{lll}
0 & 0 & 0 \\
0 & 0 & 0 \\
0 & 0 & 1
\end{array}\right), \quad(C B)^{\sharp}=C B=\left(\begin{array}{ll}
1 & 0 \\
2 & 0
\end{array}\right), \\
& (C B)^{\pi}=\left(\begin{array}{cc}
0 & 0 \\
-2 & 1
\end{array}\right),
\end{aligned}
$$

so $A^{\pi} B(C B)^{\pi}=0$. By Theorem $1, M^{\sharp}$ exists and

$$
M^{\sharp}=\left(\begin{array}{ccccc}
0 & 0 & 0 & 1 & 0 \\
7 & -1 & 0 & -13 & 3 \\
-2 & 0 & 0 & 4 & 0 \\
1 & 0 & 0 & -1 & 0 \\
2 & 0 & 0 & -2 & 0
\end{array}\right) .
$$

Similarly, we can prove the counterpart of Theorem 1.

Theorem 2 Let $M=\left(\begin{array}{ll}A & B \\ C & 0\end{array}\right)$, where $A \in \Re^{n \times n}, B \in \Re^{n \times m}, C \in \Re^{m \times n}$. If $A B=B$, then

(i) $M^{\sharp}$ exists if and only if $(C B)^{\sharp}$ and $A^{\sharp}$ exist and $(C B)^{\pi} C A^{\pi}=0$;

(ii) If $M^{\sharp}$ exists, then $M^{\sharp}=\left(\begin{array}{ll}M_{1} & M_{2} \\ M_{3} & M_{4}\end{array}\right)$, where

$$
\begin{aligned}
& M_{1}=A^{\sharp}-B(C B)^{\pi} C A^{\sharp}-B(C B)^{\pi} C\left(A^{\sharp}\right)^{2}-B(C B)^{\sharp} C A^{\sharp}-B\left[(C B)^{\sharp}\right]^{2} C A^{\pi}, \\
& M_{2}=B(C B)^{\sharp}+B(C B)^{\pi}, \\
& M_{3}=(C B)^{\sharp} C A^{\sharp}+(C B)^{\sharp} C A^{\pi}+\left[(C B)^{\sharp}\right]^{2} C A^{\pi}+(C B)^{\pi} C\left(A^{\sharp}\right)^{2}, \\
& M_{4}=-(C B)^{\sharp} .
\end{aligned}
$$

Next we consider a special case of Theorems 1 and 2, and investigate it over any ring. 
Theorem 3 Let $M=\left(\begin{array}{ll}A & B \\ C & 0\end{array}\right)$, where $A \in \mathbf{R}^{n \times n}, B \in \mathbf{R}^{n \times m}, C \in \mathbf{R}^{m \times n}$. If $A B=$ $B, C A=C$, then

(i) $M^{\sharp}$ exists if and only if $(C B)^{\sharp}$ and $A^{\sharp}$ exist;

(ii) If $M^{\sharp}$ exists, then

$$
M^{\sharp}=\left(\begin{array}{cc}
A^{\sharp}-2 B(C B)^{\pi} C-B(C B)^{\sharp} C & B(C B)^{\sharp}+B(C B)^{\pi} \\
(C B)^{\sharp} C+(C B)^{\pi} C & -(C B)^{\sharp}
\end{array}\right) .
$$

Proof (i) The "only if" part.

If $M^{\sharp}$ exists, then by Lemma 2 there exist matrices $X$ and $Y$ over $\mathbf{R}$ such that $M=M^{2} X$ and $M=Y M^{2}$.

By $A B=B$ and $C A=C$, we have

$$
\left(\begin{array}{cc}
I & 0 \\
C & I
\end{array}\right)\left(\begin{array}{cc}
A^{2} & 0 \\
-C B C & C B C B
\end{array}\right)\left(\begin{array}{cc}
I & B \\
0 & I
\end{array}\right)\left(\begin{array}{cc}
I & 0 \\
C & I
\end{array}\right)=\left(\begin{array}{cc}
A^{2}+B C & B \\
C & C B
\end{array}\right)=M^{2} .
$$

Let

$$
X=\left(\begin{array}{cc}
I & 0 \\
-C & I
\end{array}\right)\left(\begin{array}{cc}
I & -B \\
0 & I
\end{array}\right)\left(\begin{array}{ll}
X_{1} & X_{2} \\
X_{3} & X_{4}
\end{array}\right)\left(\begin{array}{ll}
I & B \\
0 & I
\end{array}\right)
$$

Then

$$
\left(\begin{array}{cc}
A^{2} & 0 \\
-C B C & C B C B
\end{array}\right)\left(\begin{array}{ll}
X_{1} & X_{2} \\
X_{3} & X_{4}
\end{array}\right)=\left(\begin{array}{cc}
A & 0 \\
0 & -C B
\end{array}\right) .
$$

From above, we get

$$
\begin{gathered}
A^{2} X_{1}=A, \\
A^{2} X_{2}=0, \\
-C B C X_{1}+C B C B X_{3}=0, \\
-C B C X_{2}+C B C B X_{4}=-C B .
\end{gathered}
$$

It is easy to get

$$
\left(\begin{array}{ll}
I & B \\
0 & I
\end{array}\right)\left(\begin{array}{cc}
I & 0 \\
C & I
\end{array}\right)\left(\begin{array}{cc}
A^{2} & B-B C B \\
0 & C B C B
\end{array}\right)=M^{2}
$$

From above equations and $M=Y M^{2}$, let $Y=\left(\begin{array}{cc}Y_{1} & Y_{2} \\ Y_{3} & Y_{4}\end{array}\right)$, we have

$$
\begin{gathered}
Y_{1} A^{2}=A, \\
Y_{1}(B-B C B)+Y_{2} C B C B=B, \\
Y_{3} A^{2}=C, \\
Y_{3}(B-B C B)+Y_{4} C B C B=0 .
\end{gathered}
$$


By Lemma 2, (6) and (10) imply $A^{\sharp}$ exists.

By (7) and (12), we have $C X_{2}=0$ and $Y_{3} B=C B$. Substitute these identities into (9) and (13) respectively, we get $C B C B X_{4}=-C B$ and $C B=\left(I-Y_{4}\right) C B C B$.

By Lemma $1,(C B)^{\sharp}$ exists.

The 'if' part. Let

$$
\begin{aligned}
& X_{1}=A^{\sharp}, X_{2}=0, X_{3}=(C B)^{\sharp} C, X_{4}=-(C B)^{\sharp} . \\
& Y_{1}=A^{\sharp}, Y_{2}=B(C B)^{\sharp}, Y_{3}=C, Y_{4}=C B(C B)^{\sharp}-(C B)^{\sharp} .
\end{aligned}
$$

It is easy to verify (6)-(13) hold. That implies $M=M^{2} X$ and $M=Y M^{2}$ have solutions.

From Lemma 2, we know $M^{\sharp}$ exists.

(ii) By Lemma 2, the expression of $M^{\sharp}$ can get from $M^{\sharp}=Y M X$.

Example for Theorem 3: Let $Z$ be the integer ring, and let $M=\left(\begin{array}{ll}A & B \\ C & 0\end{array}\right)$ be a matrix over $Z /(6 Z)$, where

$$
A=\left(\begin{array}{ccc}
1 & -2 & 0 \\
0 & 1 & 0 \\
0 & 0 & 0
\end{array}\right), \quad B=\left(\begin{array}{ll}
3 & 1 \\
3 & 3 \\
0 & 0
\end{array}\right), \quad C=\left(\begin{array}{lll}
3 & 1 & 0 \\
3 & 2 & 0
\end{array}\right) .
$$

It is easy to verify that $A^{2} \neq A, A B=B, C A=C$. Furthermore, $A^{\sharp}$ and $(C B)^{\sharp}$ exist.

By computation,

$$
A^{\sharp}=\left(\begin{array}{lll}
1 & 2 & 0 \\
0 & 1 & 0 \\
0 & 0 & 0
\end{array}\right), \quad(C B)^{\sharp}=C B=\left(\begin{array}{ll}
0 & 0 \\
3 & 3
\end{array}\right) .
$$

By Theorem 3, $M^{\sharp}$ exists and

$$
M^{\sharp}=\left(\begin{array}{ccccc}
1 & 1 & 0 & 3 & 1 \\
0 & -2 & 0 & 3 & 3 \\
0 & 0 & 0 & 0 & 0 \\
3 & 1 & 0 & 0 & 0 \\
3 & 2 & 0 & 3 & 3
\end{array}\right) .
$$

Remark 1 From Theorem 1 and 2, we can obtain Theorem 3.1 and 3.2 of [27] and Theorem 2.1 and 2.2 of [10]. From Theorem 3, we can also obtain the Corollary 2.2 of [10]. In above two examples, we especially point that $A^{2} \neq A$. This shows that the generalizations are true.

The following results extend the corresponding works of Zhao et al. [20].

Theorem 4 Let $M=\left(\begin{array}{cc}A & B \\ B & 0\end{array}\right)$, where $A, B \in \Re^{n \times n}$. If $B^{\sharp}$ exists and $B A B^{\pi}=0$. Then (i) $M^{\sharp}$ exists if and only if $\left(A B^{\pi}\right)^{\sharp}$ exists. 
(ii) If $M^{\sharp}$ exists, then $M^{\sharp}=\left(\begin{array}{ll}M_{1} & M_{2} \\ M_{3} & M_{4}\end{array}\right)$, where

$$
\begin{aligned}
& M_{1}=B^{\pi} A\left(B^{\sharp}\right)^{2}-\left(A B^{\pi}\right)^{\sharp} A B^{\pi} A\left(B^{\sharp}\right)^{2}+\left(A B^{\pi}\right)^{\sharp} ; \\
& M_{2}=-B^{\pi} A\left(B^{\sharp}\right)^{2} A B^{\sharp}+\left(A B^{\pi}\right)^{\sharp} A B^{\pi} A\left(B^{\sharp}\right)^{2} A B^{\sharp}-\left(A B^{\pi}\right)^{\sharp} A B^{\sharp}+B^{\sharp} ; \\
& M_{3}=B^{\sharp} ; \\
& M_{4}=-B^{\sharp} A B^{\sharp} .
\end{aligned}
$$

Proof The "only if" part of (i).

It is easy to get

$$
\begin{array}{cc}
M=\left(\begin{array}{cc}
A & B \\
B & 0
\end{array}\right) & =\left(\begin{array}{cc}
B^{\pi} A & B \\
B & 0
\end{array}\right)\left(\begin{array}{cc}
I & O \\
B^{\sharp} A & I
\end{array}\right), \\
M^{2}=\left(\begin{array}{cc}
A^{2}+B^{2} & A B \\
B A & B^{2}
\end{array}\right) & =\left(\begin{array}{cc}
A B^{\pi} A+B^{2} & A B \\
0 & B^{2}
\end{array}\right)\left(\begin{array}{cc}
I & O \\
B^{\sharp} A & I
\end{array}\right) .
\end{array}
$$

Since $M^{\sharp}$ exists, from Lemma 1 we know $Y M^{2}=M$ has a solution.

Let $Y=\left(\begin{array}{ll}Y_{1} & Y_{2} \\ Y_{3} & Y_{4}\end{array}\right)$.

We have

$$
\begin{gathered}
Y_{1} A B^{\pi} A+Y_{1} B^{2}=B^{\pi} A, \\
Y_{1} A B+Y_{2} B^{2}=B, \\
Y_{3} A B^{\pi} A+Y_{3} B^{2}=B, \\
Y_{3} A B+Y_{4} B^{2}=0 .
\end{gathered}
$$

By (14), we have $Y_{1}\left(A B^{\pi}\right)^{2}=B^{\pi} A B^{\pi}=A B^{\pi}$. From Lemma 1, we know $\left(A B^{\pi}\right)^{\sharp}$ exists. Next, we prove the sufficiency of (i) and the expression of (ii):

Let $X=\left(\begin{array}{ll}M_{1} & M_{2} \\ M_{3} & M_{4}\end{array}\right)$. By Lemma 3, the sufficiency of (i) and the expression of $M^{\sharp}$ are similar to the proof in [20].

$$
M X=\left(\begin{array}{cc}
A\left(A B^{\pi}\right)^{\sharp}+B B^{\sharp} & -A\left(A B^{\pi}\right)^{\sharp} A B^{\sharp}+B^{\pi} A B^{\sharp} \\
0 & B B^{\sharp}
\end{array}\right)=X M .
$$

It is easy to verify that $X M X=M, M X M=M$.

So $X=M^{\sharp}$.

Similarly, we state the symmetrical result of Theorem 4.

Theorem 5 Let $M=\left(\begin{array}{ll}A & B \\ B & 0\end{array}\right)$, where $A, B \in \Re^{n \times n}$. If $B^{\sharp}$ exists and $B^{\pi} A B=0$, then

(i) $M^{\sharp}$ exists if only if $\left(B^{\pi} A\right)^{\sharp}$ exists. 
(ii) If $M^{\sharp}$ exists, then $M^{\sharp}=\left(\begin{array}{ll}M_{1} & M_{2} \\ M_{3} & M_{4}\end{array}\right)$, where

$$
\begin{aligned}
& M_{1}=\left(B^{\sharp}\right)^{2} A B^{\pi}-\left(B^{\sharp}\right)^{2} A B^{\pi} A\left(B^{\pi} A\right)^{\sharp}+\left(B^{\pi} A\right)^{\sharp} ; \\
& M_{2}=B^{\sharp} ; \\
& M_{3}=-B^{\sharp} A\left(B^{\sharp}\right)^{2} A B^{\pi}+B^{\sharp} A\left(B^{\sharp}\right)^{2} A B^{\pi} A\left(B^{\pi} A\right)^{\sharp}-B^{\sharp} A\left(B^{\pi} A\right)^{\sharp}+B^{\sharp} ; \\
& M_{4}=-B^{\sharp} A B^{\sharp} .
\end{aligned}
$$

Proof The proof is similar to Theorem 4, so we omit it.

Next we consider a special case of Theorem 4 and 5, and investigate it over any ring.

Theorem 6 Let $M=\left(\begin{array}{cc}A & B \\ B & 0\end{array}\right)$, where $A, B \in \mathbf{R}^{n \times n}$. If $B^{\sharp}$ exists and $B A B^{\pi}=$ $0, B^{\pi} A B=0$, then

(i) $M^{\sharp}$ exists if and only if $\left(A B^{\pi}\right)^{\sharp}$ exists.

(ii) If $M^{\sharp}$ exists, then

$$
M^{\sharp}=\left(\begin{array}{cc}
\left(A B^{\pi}\right)^{\sharp} & B^{\sharp} \\
B^{\sharp} & -B^{\sharp} A B^{\sharp}
\end{array}\right) .
$$

Proof The "only if" part of (i).

Let

$$
M^{2}=\left(\begin{array}{cc}
I & A B^{\sharp} \\
0 & I
\end{array}\right)\left(\begin{array}{cc}
A B^{\pi} A+B^{2} & 0 \\
0 & B^{2}
\end{array}\right)\left(\begin{array}{cc}
I & O \\
B^{\sharp} A & I
\end{array}\right) .
$$

The decomposition of $M$ is the same as in Theorem 4. By Lemma 2, $M^{\sharp}$ exists if and only if there exist $X, Y$ over $\mathbf{R}$ such that $M X^{2}=M$ and $Y M^{2}=M$.

Let

$$
X=\left(\begin{array}{cc}
I & 0 \\
-B^{\sharp} A & I
\end{array}\right)\left(\begin{array}{cc}
X_{1} & X_{2} \\
X_{3} & X_{4}
\end{array}\right), \quad Y=\left(\begin{array}{ll}
Y_{1} & Y_{2} \\
Y_{3} & Y_{4}
\end{array}\right)\left(\begin{array}{cc}
I & -A B^{\sharp} \\
0 & I
\end{array}\right),
$$

then we have

$$
\begin{gathered}
A B^{\pi}=\left(A B^{\pi} A+B^{2}\right) X_{1}, \\
B=\left(A B^{\pi} A+B^{2}\right) X_{2}, \\
B=B^{2} X_{3}, \\
0=B^{2} X_{4}, \\
Y_{1} A B^{\pi} A+Y_{1} B^{2}=B^{\pi} A, \\
Y_{2} B^{2}=B, \\
Y_{3} A B^{\pi} A+Y_{3} B^{2}=B, \\
Y_{4} B^{2}=0 .
\end{gathered}
$$


By (18) and (22), we have $A B^{\pi} A B^{\pi} X_{1}=B^{\pi} A B^{\pi} A B^{\pi} X_{1}=B^{\pi} A B^{\pi} A X_{1}=$ $B^{\pi} A B^{\pi}=A B^{\pi}$ and $Y_{1} A B^{\pi} A B^{\pi}=A B^{\pi}$, respectively. From Lemma 2, we know $\left(A B^{\pi}\right)^{\sharp}$ exists. Next, we prove the sufficiency of (i) and the expression of (ii).

Let

$$
X=\left(\begin{array}{cc}
\left(A B^{\pi}\right)^{\sharp} & B^{\sharp} \\
B^{\sharp} & -B^{\sharp} A B^{\sharp}
\end{array}\right) .
$$

By Lemma 3,

$$
M X=\left(\begin{array}{cc}
A\left(A B^{\pi}\right)^{\sharp}+B B^{\sharp} & 0 \\
0 & B B^{\sharp}
\end{array}\right)=X M .
$$

It is easy to verify that $X M X=M, M X M=M$.

So $X=M^{\sharp}$.

Remark 2 We have expressed Theorems 1-2 and 4-5 over right Ore domains, but how to solve them in rings? This is still an open question.

Acknowledgements The authors would like to thank the editors and the referees for their very detailed comments and valuable suggestions which greatly improved our paper.

Open Access This article is distributed under the terms of the Creative Commons Attribution License which permits any use, distribution, and reproduction in any medium, provided the original author(s) and the source are credited.

\section{References}

1. Campbell, S.L., Meyer, C.D., Rose, N.J.: Application of the Drazin inverse to linear system of differential equations with singular constant coefficients. SIAM J. Appl. Math. 24, 45-49 (1976)

2. Soares, A.S., Latouche, G.: The group inverse of finite homogeneous QBD processes. Stoch. Models 18, 159-171 (2002)

3. Wei, Y., Diao, H.: On group inverse of singular Toeplitz matrices. Linear Algebra Appl. 399, 109-123 (2005)

4. Ben-Israel, A., Greville, T.N.E.: Generalized Inverse: Theory and Applications, 2nd edn. Wiley, New York (1974)

5. Climent, J.J., Neumann, M., Sidi, A.: A semi-iterative method for real spectrum singular liner systems with an arbitrary index. J. Comput. Appl. Math. 87, 21-38 (1997)

6. Wang, G.: A Cramer rule for finding the solution of a class of singular equations. Linear Algebra Appl. 116, 27-34 (1980)

7. Golub, G.H., Greif, C.: On solving block-structured indefinite linear systems. SIAM J. Sci. Comput. 24, 2076-2092 (2003)

8. Wei, Y.: Index splitting for the Drazin inverse and the singular linear system. Appl. Math. Comput. 95, 115-124 (1998)

9. Campbell, S.L.: The Drazin inverse and systems of second order linear differential equations. Linear Multilinear Algebra 14, 195-198 (1983)

10. Liu, X., Yang, H.: Further results on the group inverses and Drazin inverses of anti-triangular block matrices. Appl. Math. Comput. 218, 8978-8986 (2012)

11. Bhaskara Rao, K.P.S.: The Theory of Generalized Inverses over Commutative Rings. Taylor and Francis, London and New York (2002)

12. Zhang, K., Bu, C.: Group inverses of matrices over right Ore domains. Appl. Math. Comput. 218, 6942-6953 (2012)

13. Cao, C., Zhao, C.: Group inverse for a class of $2 \times 2$ anti-triangular block matrices over skew fields. J. Appl. Math. Comput. 40, 87-93 (2012) 
14. Bu, C., Zhao, J., Zhang, K.: Some results on group inverses of block matrices over skew fields. Electron. J. Linear Algebra 18, 117-125 (2009)

15. Bu, C., Zhao, J., Zheng, J.: Group inverse for a class $2 \times 2$ block matrices over skew fields. Appl. Math. Comput. 204, 45-49 (2008)

16. Cao, C., Li, J.: Group inverses for matrices over a Bézout domain. Electron. J. Linear Algebra 18, 600-612 (2009)

17. Zhao, J., Hu, Z., Zhang, K., Bu, C.: Group inverse for block matrix with t-potent subblock. J. Appl. Math. Comput. 39, 109-119 (2012)

18. Cao, C., $\mathrm{Li}$, J.: A note on the group inverse of some $2 \times 2$ block matrices over skew fields. Appl. Math. Comput. 217, 10271-10277 (2011)

19. Patrício, P., Hartwig, R.E.: The (2, 2, 0) group inverse problem. Appl. Math. Comput. 217, 516-520 (2010)

20. Zhao, J., Bu, C.: Group inverse for the block matrix with two identical subblocks over skew fields. Electron. J. Linear Algebra 21, 63-75 (2010)

21. Bu, C., Zhang, K., Zhao, J.: Some results on the group inverse of the block matrix with a sub-block of linear combination or product combination of matrix over skew fields. Linear Multilinear Algebra 58, 959-966 (2010)

22. Cao, C., Tang, X.: Representation of the group inverse of some $2 \times 2$ block matrices. Int. Math. Forum 31, 1511-1517 (2006)

23. Cohn, P.M.: Free Rings and Their Relations, 2nd edn. London Mathematical Society Monographs, vol. 9. Academic Press, London (1985)

24. Zhuang, W.: The Guidance of Matrix Theory over Skew Fields. Science Press, Beijing (2006) (in Chinese)

25. Huang, L.: Geometry of Matrices over Ring. Science Press, Beijing (2006)

26. Sheng, Y., Ge, Y., Zhang, H., Cao, C.: Group inverse for a class of $2 \times 2$ block matrices over rings. Appl. Math. Comput. 219, 9340-9346 (2013)

27. Ge, Y., Zhang, H., Sheng, Y., Cao, C.: Group inverse for two classes of $2 \times 2$ anti-triangular block matrices over right Ore domains. J. Appl. Math. Comput. 42, 183-191 (2013) 